\title{
GLOBAL INVARIANTS FOR STRONGLY PSEUDOCONVEX VARIETIES WITH ISOLATED SINGULARITIES: BERGMAN FUNCTIONS
}

\author{
STEPhen S.-T. YAU
}

\begin{abstract}
Let $M$ be a strongly pseudoconvex manifold which is a resolution of strongly pseudoconvex variety $V$ with only isolated singularities. We define a Bergman function $B_{M}$ on $M$ which is a biholomorphic invariant of $M$. The Bergman function $B_{M}$ vanishes precisely on the exceptional set of $M$. Hence $B_{M}$ can be pushed down and we obtain a Bergman function $B_{V}$ which is a biholomorphic invariant of $V$ and vanishes precisely on the singularities of $V$. This Bergman function not only can distinguish analytic structures of isolated singularities, but it can also distinguish the $\mathrm{CR}$ structures of the boundaries of $V$. As an application, we define a continuous numerical invariant on strongly pseudoconvex $\mathrm{CR}$ manifolds in $V=\left\{(x, y, z) \in \mathbb{C}^{3}: x y=z^{2}\right\}$. We show that our invariant varies continuously in $\mathbb{R}$ when the CR structure of strongly pseudoconvex CR manifold changes in $V$. Our global numerical invariant is explicitly computable. Moreover we show that the Bergman function allows us to determine the automorphism groups of these CR manifolds.
\end{abstract}

\section{Introduction}

The Bergman kernel form is a basic biholomorphic invariant on complex manifolds [Ko]. A lot of work has been done in its explicit computation and asymptotic expansion. However, it seems that there is little attention given to the possible role of the Bergman kernel on analytic spaces, in connection with the study of singularities and CR manifolds. In [L-Y-Y], an initial step in studying the Bergman kernel on a resolution of an isolated 2-dimensional Gorenstein singularity was given. It was shown that the exceptional set of the resolution is exactly the minimal set of the Bergman kernel. Thus the analytic definition of the Bergman kernel contains important topological information on the singularity. However, the Bergman kernel defined in $[\mathrm{L}-\mathrm{Y}-\mathrm{Y}]$ is not a biholomorphically invariant except for the rational double points.

Let $M$ be a complex manifold of dimension $n$. A real valued $C^{\infty}$ function $\phi$ on $M$ is said to be strongly plurisubharmonic if and only if the hermitian form

$$
\sum_{i, j=1}^{n} \frac{\partial^{2} \phi}{\partial z_{i} \partial \overline{z_{j}}} d z_{i} \overline{d z_{j}}
$$

Received February 13, 2004.

Research partially supported by NSF and by Hong Kong RGC.. 
is positive definite with respect to any system of local coordinates $\left(z_{1}, \ldots, z_{n}\right)$. The complex manifold $M$ is said to be strongly pseudoconvex if there is a compact subset $B \subset M$, and a continuous real valued function $\phi$ on $M$, which is strongly plurisubharmonic outside $B$, and such that for each $c \in \mathbb{R}$, the set $B_{c}=\{x \in M: \phi(x)<c\}$ is relatively compact in $M$. Note that a strongly pseudoconvex manifold is a modification of a Stein space at a finite many points.

The purpose of this paper is to define a new Bergman function $B_{M}$ for each strongly pseudoconvex manifold $M$ with dimension $n \geq 2$ which is a resolution of strongly pseudoconvex variety $V$ with only isolated singularities. We show that $B_{M}$ is a biholomorphically invariant function and $B_{M}$ vanishes precisely on the exceptional set of $M$. Hence $B_{M}$ can be pushed down and we obtain a Bergman function $B_{V}$ which is a biholomorphic invariant of $V$ and vanishes precisely on the singularities of $V$.

The invariance properties of a CR manifold $X$ of real dimension $2 n-1$ which is a real hypersurface in $\mathbb{C}^{n}$ with respect to the infinite pseudo-group of biholomorphic transformations were studied extensively by many important mathematicians. The systematic study of such properties for hypersurfaces with nondegenerate Levi form was first made by Catan [Ca] in 1932, and latter by Chern and Moser [Ch-Mo]. A main result of the theory is the existence of a complete system of local differential invariants. On the other hand, by using the Catan method of equivalence, Webster [We] gave a complete characterization when two ellipsoids in $\mathbb{C}^{n}$ are $\mathrm{CR}$ equivalent. In [Fe], Fefferman has shown that a biholomorphic map between two bounded strongly pseudoconvex domains with smooth boundaries extends smoothly to the boundaries. Then Webster's result gives a necessary and sufficient condition for two ellipsoidal domains to be equivalent.

Despite the success of the Chern-Moser theory, the fundamental question of distinguishing two strongly pseudoconvex manifolds remains unsolved. Let $X$ be a compact connected strongly pseudoconvex CR manifold of real dimension $2 n-1$. In 1974, Boutel de Monvel [Bo] (see also Kohn [Koh]) proved that $X$ is CR-embeddable in some $\mathbb{C}^{N}$ if $\operatorname{dim} X \geq 5$. In this paper, we shall only consider CR embeddable strongly pseudoconvex CR manifolds. Let $X$ be an embeddable compact strongly pseudoconvex $\mathrm{CR}$ manifold. In view of a beautiful theorem of Harvey-Lawson [Ha-La], there exists a complex variety $V$ in $\mathbb{C}^{N}$ such that $\partial V=X$ and $V$ has only normal isolated singularities. Theorem 3.1 below says that we can use the structures of the singularities of $V$ to distinguish the CR structure of $X$. Thus if two strongly pseudoconvex manifolds bound nonisomorphic singularities, then their CR structures are different. The difficult unsolved CR equivalence problem is: how can one distinguish strongly pseudoconvex CR manifolds $X_{1}$ and $X_{2}$ when they are lying in the same variety $V$. If $V$ is $\mathbb{C}^{N}$, this difficult problem has been considered by leading mathematicians Chern-Moser [Ch-Mo], Fefferman [Fe], Webster [We], etc. Even in this case, it seems that the CR equivalence problem for complete Reinhardt domains (except for the ellipsoidal domains which was solved by Webster) remains open. On the 
other hand, when $V$ is a singular variety, the CR equivalence problem is basically untouched. One of the purpose of this paper is to offer a novel technique to attack CR equivalence problem. The main observation is that our new Bergman functions put a lot of restriction on biholomorphic maps between strongly pseudoconvex CR manifolds, from which new CR invariants can be constructed and the automorphism groups of the CR manifolds can be determined. We illustrate how our new technique works in a concrete example.

We define a continuous numerical invariant on strongly pseudoconvex CR manifolds in $V=\left\{(x, y, z) \in \mathbb{C}^{2}: x y=z^{2}\right\}$. We show that our invariant varies continuously in $\mathbb{R}$ when the $\mathrm{CR}$ structure of strongly pseudoconvex $\mathrm{CR}$ manifold changes in $V$. Our global numerical invariant is explicitly computable. Moreover we show that the Bergman function allows us to determine the automorphism groups of these CR manifolds.

We thank the referee for many useful suggestions to improve the presentation of this paper.

\section{Bergman function on strongly pseudoconvex manifold and variety}

Let $M$ be a complex $n$-dimensional manifold. We first recall the definition of the Bergman kernel. Let $F$ be the set of all holomorphic $n$-forms $\phi$ on $M$ such that $\left|\int_{M} \phi \wedge \bar{\phi}\right|<\infty$. ( $\phi$ will be called $L^{2}$ or square integrable.) $F$ is a separable complex Hilbert space under the inner product $\left\langle\phi_{1}, \phi_{2}\right\rangle=(\sqrt{-1})^{n^{2}} \int_{M} \phi_{1} \wedge$ $\bar{\phi}_{2}$. The corresponding norm $\langle\phi, \phi\rangle^{\frac{1}{2}}$ will be denoted by $\|\phi\|$. Let $\left\{\omega_{j}\right\}$ be a complete orthonormal basis of $F$. Then $K(z, \bar{w})=\Sigma \omega_{j}(z) \wedge \overline{\omega_{j}(w)}$ can be shown to converge uniformly on compact subsets to a holomorphic $2 n$-form on $M \times \bar{M}$. Here, $\bar{M}$ denotes the conjugate complex manifold obtained by taking the conjugate coordinate charts of $M$. Further, $K(z, \bar{w})$ is independent of the choice of complete orthonormal basis of $F$. If each point $z \in M$ is identified with the point $(z, \bar{z}) \in M \times \bar{M}$, then $K(z, \bar{z})$ can be regarded as a $2 n$-form on $M$ and is referred to as the Bergman kernel of $M$. Since the Hilbert space $F$ with its inner product is invariant under biholomorphic maps, so is the Bergman kernel.

Let $V$ be a Stein variety of dimension $n \geq 2$ in $\mathbb{C}^{N}$ with only irreducible isolated singularities. We assume that $\partial V$ is a strongly pseudoconvex CR manifold. Let $\pi: M \rightarrow V$ be a resolution of singularity with $E$ as a exceptional set. We shall define a Bergman function $B_{M}(z)$ on $M$ which is a biholomorphic invariant of $M$.

Definition 2.1. Let $F_{0}$ be the set of all $L^{2}$ integrable holomorphic $n$-forms $\psi$ on $M$ vanishing on the exception set $E$ of $M$. Let $\left\{\omega_{j}\right\}$ be a complete orthonormal basis of $F_{0}$. The Bergman kernel vanishing on the exceptional set is defined to be $K_{0}(z, \bar{z})=\Sigma \omega_{j}(z) \wedge \overline{\omega_{j}(z)}$.

Lemma 2.2. $F / F_{0}$ is a finite dimensional vector space. 
Proof. Let $\Omega^{n}$ be the sheaf of germs of holomorphic $n$-forms on $M$ and $\Omega^{n}(-E)$ be the sheaf of germs of holomorphic $n$-forms on $M$ vanishing along $E$. Clearly $F=\Gamma\left(M, \Omega^{n}\right)$ and $F_{0}=\Gamma\left(M, \Omega^{n}(-E)\right)$. From the short exact sequence

$$
\left.0 \longrightarrow \Omega^{n}(-E) \longrightarrow \Omega^{n} \longrightarrow \Omega^{n}\right|_{E} \longrightarrow 0
$$

we have $\operatorname{dim} F / F_{0} \leq \operatorname{dim} H^{0}\left(E,\left.\Omega^{n}\right|_{E}\right)$. Since $\left.\Omega^{n}\right|_{E}$ is a coherent sheaf on a compact analytic set $E, \operatorname{dim} H^{0}\left(E,\left.\Omega^{n}\right|_{E}\right)$ is finite.

Lemma 2.3. Bergman kernel vanishing on the exceptional set $K_{0}(z, \bar{z})$ is independent of the choice of the complete orthonormal basis of $F_{0}$ and $K_{0}(z, \bar{z})$ is invariant under biholomorphic maps.

Proof. Let $\left\{\omega_{i}\right\}$ and $\left\{\widetilde{\omega}_{i}\right\}$ be two complete orthonormal bases of $F_{0}$. In view of Lemma 2.2, there exists $\alpha_{1}, \ldots, \alpha_{k}$ holomorphic $n$-forms on $M$ where $k=$ $\operatorname{dim}\left(F / F_{0}\right)$ such that both $\left\{\alpha_{1}, \ldots, \alpha_{k}\right\} \cup\left\{\omega_{i}\right\}$ and $\left\{\alpha_{1}, \ldots, \alpha_{k}\right\} \cup\left\{\widetilde{\omega}_{i}\right\}$ form complete orthonormal basis of $F$. Since

$$
\sum_{i=1}^{k} \alpha_{i} \wedge \overline{\alpha_{i}}+\sum \omega_{i} \wedge \overline{\omega_{i}}=K(z, \bar{z})=\sum_{i=1}^{k} \alpha_{i} \wedge \overline{\alpha_{i}}+\sum \widetilde{\omega_{i}} \wedge \overline{\widetilde{\omega}_{i}},
$$

we have $\sum \omega_{i} \wedge \overline{\omega_{i}}=\sum \widetilde{\omega_{i}} \wedge \overline{\widetilde{\omega}_{i}}$.

Recall that exceptional set $E$ is the maximal compact analytic set in $M$. Since $E$ is invariant under biholomorphic maps, so is the space $F_{0}$ of all $L^{2}$-integrable holomorphic $n$-forms on $M$ vanishing on the exceptional set $E$. Hence $K_{0}(z, \bar{z})$ is invariant under biholomorphic maps.

Definition 2.4. Let $M$ be a resolution of a strongly pseudoconvex variety $V$ of $\operatorname{dim} n \geq 2$ in $\mathbb{C}^{N}$ with only irreducible isolated singularity at the origin. The Bergman function $B_{M}$ on $M$ is defined to be $K_{M, 0} / K_{M}$.

Theorem 2.5. $B_{M}$ is a global function defined on $M$ which is invariant under biholomorphic maps. Moreover, the zero set of $B_{M}$ is precisely the exceptional set of $M$.

Proof. Let $\Phi: M^{\prime} \longrightarrow M$ be a biholomorphic map. Then

$$
\Phi^{*}\left(B_{M}\right)=\frac{\Phi^{*} K_{M, 0}}{\Phi^{*} K_{M}}=\frac{K_{M^{\prime}, 0}}{K_{M^{\prime}}}=B_{M^{\prime}} .
$$

We first observe that there exists a holomorphic $n$-form which does not vanish on the exceptional set of $M$. This can be seen as follows. Let $\pi: M \rightarrow V$ be the blowing down map. Since $V$ is a Stein space and $\Omega_{V}^{n}$, the sheaf of germs of holomorphic $n$-forms on $V$, is coherent. There exists a holomorphic $n$-form $\omega$ on $V$ which does not vanish at the singularity set of $V$. Then $\pi^{*} \omega$ 
is a holomorphic $n$-form which does not vanish on the exceptional set of $M$. In particular, $K_{M}$ does not vanish on the exceptional set of $M$. Clearly $K_{M, 0}$ vanishes along exceptional set. To finish the proof, we need to show that $K_{M, 0}$ does not vanish outside the exceptional set. For this purpose, given $p \in M-E$, it suffices to produce a holomorphic $n$-form vanishing along $E$ but not at $p$. Let $\Omega^{n}(-E)$ be the sheaf of germs of holomorphic $n$-forms on $M$ vanishing along $E$. Since $\Omega^{n}(-E)$ is coherent and $\pi$ is proper, $\pi_{*} \Omega^{n}(-E)$ is a coherent sheaf on $V$ by Grauert's direct image theorem. As $V$ is a Stein variety, we can find $\omega$ in $\Gamma\left(V, \pi_{*} \Omega^{n}(-E)\right)$ which does not vanish at $\pi(p)$. Then $\pi^{*} \omega$ is a holomorphic $n$-form vanishing along the exceptional set $E$ but not at $p$.

The same argument of the proof of Theorem 1 in $[\mathrm{L}-\mathrm{Y}-\mathrm{Y}]$ will prove the following theorem.

Theorem 2.6. Let $M$ be a strongly pseudoconvex manifold of dimension $n \geq 2$ with exceptional set $E$. Let $A$ be compact submanifold containing in $E$. Let $\pi$ : $M_{1} \rightarrow M$ be the blow up of $M$ along $A$. Then we have $K_{M_{1}}(z, \bar{z})=\pi^{*} K_{M}(z, \bar{z})$ and $K_{M_{1}, 0}(z, \bar{z})=\pi^{*} K_{M, 0}(z, \bar{z})$. Consequently $B_{M_{1}}(z)=\pi^{*} B_{M}(z)$.

Let $\pi_{i}: M_{i} \longrightarrow V, i=1,2$, be two resolutions of singularities of $V$. By Hironaka's theorem [Hi], there exists a resolution $\widetilde{\pi}: \widetilde{M} \longrightarrow V$ of singularities of $V$ such that $\widetilde{M}$ can be obtained from $M_{i}, i=1,2$, by successive blowing up along submanifolds in exceptional set. In view of Theorem 2.5 and Theorem 2.6, the following definition is well defined.

Definition 2.7. Let $V$ be a strongly pseudoconvex variety in $\mathbb{C}^{N}$ with only irreducible isolated singularities. Let $\pi: M \rightarrow V$ be a resolution of singularities of $V$. Define the Bergman function $B_{V}$ on $V$ to be the push forward of the Bergman function $B_{M}$ by the map $\pi$.

Theorem 2.8. Let $V$ be a strongly pseudoconvex variety in $\mathbb{C}^{N}$ with only irreducible isolated singularities. Then the Bergman function $B_{V}$ on $V$ is invariant under biholomorphic maps and $B_{V}$ vanishes precisely on the singular set of $V$.

Proof. Easy consequence of Theorem 2.5, and Theorem 2.6.

Theorem 2.9. Let $V$ be a strongly pseudoconvex variety in $\mathbb{C}^{N}$ with only isolated normal singularities of dimension $n \geq 2$. Let $F_{V}$ be the set of all $L^{2}$ integrable holomorphic n-forms on $V-S$, where $S$ is the singular part of $V$. Let $F_{V, 0}=\left\{\omega \in F_{V}: \omega\right.$ vanishes on $\left.S\right\}$. Let $K_{V}(z, \bar{z})$ and $K_{V, 0}(z, \bar{z})$ be defined in the usual manner (cf. Definition 2.1). Then $B_{V}=\frac{K_{V}(z, \bar{z})}{K_{V, 0}(z, \bar{z})}$ and $B_{V}$ is a biholomorphical invariant of $V$. 
Proof. Let $\pi: M \rightarrow V$ be a resolution of singularities of $V$. It is well known (cf. [La], [Ya]) that a holomorphic $n$-form $\omega$ on a deleted neighborhood of the singular set $S$ is $L^{2}$-integrable if and only if $\pi^{*} \omega$ is a holomorphic $n$-form on a neighborhood of the exceptional set $E$ in $M$. Thus $\pi^{*}: F_{V} \rightarrow F_{M}$ is an isomorphism which sends $F_{V, 0}$ onto $F_{M, 0}$. The theorem follows easily.

\section{Continuous numerical invariant of strongly pseudoconvex CR manifold}

Let $X$ be a strongly pseudoconvex CR manifold of real dimension $2 n-1$. It is well known [Bo] that $X$ can be $\mathrm{CR}$ embedded into $\mathbb{C}^{N}$ if $n \geq 3$. For our subsequent discussion, we shall assume that $X$ is of dimension $2 n-1$ in $\mathbb{C}^{N}$. By a theorem of Harvey and Lawson [Ha-La], $X$ is a boundary of a variety $V$ with only isolated normal singularities.

Theorem 3.1. Let $X_{1}, X_{2}$ be two strongly pseudoconvex $C R$ manifolds of dimension $2 n-1$ which bound varieties $V_{1}, V_{2}$ respectively in $\mathbb{C}^{N}$ with only isolated normal singularities. If $\Phi: X_{1} \rightarrow X_{2}$ is a CR-isomorphism, then $\Phi$ can be extended to a biholomorphic map from $V_{1}$, to $V_{2}$.

Proof. Let $\phi_{1}, \ldots, \phi_{N}$ be the component functions of $\Phi$. Then $\phi_{i}$ as CR holomorphic function on $X$ can be extended in a one sided neighborhood of $X_{1}$ in $V_{1}$. By Andreotti and Grauert [An-Gr, Théoréme 15], $\phi_{i}$ can be extended holomorphically to $V_{1}-S_{1}$ where $S_{1}$ is the singular set of $V$. Since $S_{1}$ consists of only isolated normal singularities, $\phi_{i}$ can be extended holomorphically to $V_{1}$. Clearly $\left(\phi_{1}, \ldots, \phi_{N}\right)\left(V_{1}\right)$ is a variety with boundary equal to $X_{2}$. By uniqueness of complex Plateau problem, we have $\left(\phi_{1}, \ldots, \phi_{N}\right)\left(V_{1}\right)=V_{2}$.

Let $\psi_{1}, \ldots, \psi_{N}$ be the component functions of $\Psi$ which is the inverse mapping of $\Phi$. The argument above shows that $\psi_{1}, \ldots, \psi_{N}$ can be extended holomorphically to $V_{2}$ and $\left(\psi_{1}, \ldots, \psi_{N}\right)\left(V_{2}\right)=V_{1}$. Since $\left(\phi_{1}, \ldots, \phi_{N}\right) \circ\left(\psi_{1}, \ldots, \psi_{N}\right)$ restrict to $X_{1}$ is the identity map, it follows that $\left(\phi_{1}, \ldots, \phi_{N}\right) \circ\left(\psi_{1}, \ldots, \psi_{N}\right)$ is the identity map on $V_{1}$.

In view of the above Theorem 3.1, if $X_{1}$ and $X_{2}$ are two strongly pseudoconvex CR manifolds which bound varieties $V_{1}$ and $V_{2}$ with non-isomorphic singularities, then $X_{1}$ and $X_{2}$ are not CR equivalent. Therefore to study the CR equivalence of two strongly pseudoconvex CR manifolds $X_{1}$ and $X_{2}$, it remains to consider the case when $X_{1}$ and $X_{2}$ are lying on the same variety $V$. The purpose of this section is to show that our global invariant Bergman function defined in section 2 can be used to study the CR equivalence problem of strongly pseudoconvex CR manifolds lying on the same variety. As an example, we shall show explicitly that how CR manifolds varies in the variety $\widetilde{V}:=\left\{(x, y, z) \in \mathbb{C}^{3}: f(x, y, z)=\right.$ $\left.x y-z^{2}=0\right\}$. An explicit resolution $\widetilde{\pi}: \widetilde{M} \rightarrow \widetilde{V}$ can be given in terms of 
coordinate charts and transition functions as follows:

$$
\begin{aligned}
\text { Coordinate charts } & : \widetilde{W}_{k}=\mathbb{C}^{2}=\left\{\left(u_{k}, v_{k}\right)\right\}, k=0,1 \\
\text { Transition functions } & :\left\{\begin{array} { c } 
{ u _ { 1 } = \frac { 1 } { v _ { 0 } } } \\
{ v _ { 1 } = u _ { 0 } v _ { 0 } ^ { 2 } }
\end{array} \text { or } \left\{\begin{array}{c}
u_{0}=u_{1}^{2} v_{1} \\
v_{0}=\frac{1}{u_{1}}
\end{array}\right.\right. \\
\text { Resolution map }: & \tilde{\pi}\left(u_{k}, v_{k}\right)=\left(u_{k}^{k+1} v_{k}^{k}, u_{k}^{1-k} v_{k}^{2-k}, u_{k} v_{k}\right) \text { or } \\
(x, y, z)= & \left(u_{0}, u_{0} v_{0}^{2}, u_{0} v_{0}\right)=\left(u_{1}^{2} v_{1}, v_{1}, u_{1} v_{1}\right)
\end{aligned}
$$$$
\text { Exceptional set } \quad: \quad E=\pi^{-1}(0)=C_{1}=\left\{u_{0}=0\right\} \cup\left\{v_{1}=0\right\} .
$$

We consider $V=\left\{(x, y, z) \in \mathbb{C}^{3}: x y=z^{2}\right.$ and $\eta(x, y, z)<\epsilon_{0}$, where $\eta$ is a strictly plurisubharmonic function $\}$. Then $M=\widetilde{\pi}^{-1}(V)$ is given by the coordinate charts:

$$
W_{k}=\left\{\left(u_{k}, v_{k}\right): \eta\left(u_{k}^{k+1} v_{k}^{k}, u_{k}^{1-k} v_{k}^{2-k}, u_{k} v_{k}\right)<\epsilon_{0}\right\}, \quad k=0,1 .
$$

Observe that under $\pi: M \rightarrow V, W_{0} \backslash C_{1}$ is mapped biholomorphically onto $V \backslash y$-axis. In particular $M \backslash W_{0}$ is of measure zero in the obvious sense. Hence, we may compute integrals on $M$ using the $\left(u_{0}, v_{0}\right)$ coordinate on the chart $W_{0}$ alone.

In what follows, we shall assume that $\eta$ is a Reinhardt function such that $W_{0}$ is a complete Reinhardt domain, i.e. whenever $\left(u_{0}, v_{0}\right) \in W_{0}$, then $\left(\tau_{1} u_{0}, \tau_{2}, v_{0}\right) \in$ $W_{0}$ for all complex numbers $\tau_{j}$ with $\left|\tau_{j}\right| \leq 1$. The following proposition can be found in Proposition 8 of [L-Y-Y].

Proposition 3.2. In the above notations, let $\phi_{\alpha \beta}=u_{0}^{\alpha} v_{0}^{\beta} d u_{0} \wedge d v_{0}, \alpha, \beta=$ $0,1,2, \ldots$ Assume that $W_{0}$ is a complete Reinhardt domain. Then

$$
\left\{\frac{\phi_{\alpha \beta}}{\left\|\phi_{\alpha \beta}\right\|_{M}}: \alpha \geq \frac{1}{2} \beta\right\}
$$

is a complete orthonormal base of $F$. In other words, a complete orthonormal base of $F$ is of the form:

$$
\left\{\begin{array}{l}
\frac{1}{\left\|\phi_{00}\right\|_{M}} d u_{0} \wedge d v_{0}, \quad \frac{u_{0}}{\left\|\phi_{10}\right\|_{M}} d u_{0} \wedge d v_{0}, \quad \frac{u_{0} v_{0}}{\left\|\phi_{11}\right\|_{M}} d u_{0} \wedge d v_{0}, \\
\frac{u_{0} v_{0}^{2}}{\left\|\phi_{12}\right\|_{M}} d u_{0} \wedge d v_{0}, \quad \frac{u_{0}^{2}}{\left\|\phi_{20}\right\|_{M}} d u_{0} \wedge d v_{0}, \quad \frac{u_{0}^{2} v_{0}}{\left\|\phi_{21}\right\|_{M}} d u_{0} \wedge d v_{0}, \quad \frac{u_{0}^{2} v_{0}^{2}}{\left\|\phi_{22}\right\|_{M}} d u_{0} \wedge d v_{0}, \\
\frac{u_{0}^{2} v_{0}^{3}}{\left\|\phi_{23}\right\|_{M}} d u_{0} \wedge d v_{0}, \quad \frac{u_{0}^{2} v_{0}^{4}}{\left\|\phi_{24}\right\|_{M}} d u_{0} \wedge d v_{0}, \frac{u_{0}^{3}}{\left\|\phi_{30}\right\|_{M}} d u_{0} \wedge d v_{0}, \frac{u_{0}^{3} v_{0}}{\left\|\phi_{31}\right\|_{M}} d u_{0} \wedge d v_{0}, \\
\frac{u_{0}^{3} v_{0}^{2}}{\left\|\phi_{32}\right\|_{M}} d u_{0} \wedge d v_{0}, \quad \frac{u_{0}^{3} v_{0}^{3}}{\left\|\phi_{33}\right\|_{M}} d u_{0} \wedge d v_{0}, \quad \frac{u_{0}^{3} v_{0}^{4}}{\left\|\phi_{34}\right\|_{M}} d u_{0} \wedge d v_{0}, \frac{u_{0}^{3} v_{0}^{5}}{\left\|\phi_{35}\right\|_{M}} d u_{0} \wedge d v_{0}, \\
\frac{u_{0}^{3} v_{0}^{6}}{\left\|\phi_{36}\right\|_{M}} d u_{0} \wedge d v_{0}, \ldots
\end{array}\right\}
$$


Observe that except for $\frac{1}{\left\|\phi_{00}\right\|} d u_{0} \wedge d v_{0}$, all the other holomorphic 2-forms above are vanishing at the exceptional set. Therefore the Bergman kernel vanishing on the exceptional set $K_{M, 0}$ and Bergman kernel are given respectively by:

$$
\begin{aligned}
K_{M, 0}\left(\left(u_{0}, v_{0}\right),\left(\bar{u}_{0}, \bar{v}_{0}\right)\right) & =\Theta_{M} d u_{0} \wedge d v_{0} \wedge d \overline{u_{0}} \wedge d \overline{v_{0}} \\
K_{M}\left(\left(u_{0}, v_{0}\right),\left(\overline{u_{0}}, \overline{v_{0}}\right)\right) & =\left(\frac{1}{\left\|\phi_{00}\right\|_{M}^{2}}+\Theta_{M}\right) d u_{0} \wedge d v_{0} \wedge d \overline{u_{0}} \wedge d \overline{v_{0}}
\end{aligned}
$$

where

$$
\begin{aligned}
\Theta_{M}= & \frac{\left|u_{0}\right|^{2}}{\left\|\phi_{10}\right\|_{M}^{2}}+\frac{\left|u_{0}\right|^{2}\left|v_{0}\right|^{2}}{\left\|\phi_{11}\right\|_{M}^{2}}+\frac{\left|u_{0}\right|^{2}\left|v_{0}\right|^{4}}{\left\|\phi_{12}\right\|_{M}^{2}}+\frac{\left|u_{0}\right|^{4}}{\left\|\phi_{20}\right\|_{M}^{2}}+\frac{\left|u_{0}\right|^{4}\left|v_{0}\right|^{2}}{\left\|\phi_{21}\right\|_{M}^{2}} \\
& +\frac{\left|u_{0}\right|^{4}\left|v_{0}\right|^{4}}{\left\|\phi_{22}\right\|_{M}^{2}}+\frac{\left|u_{0}\right|^{4}\left|v_{0}\right|^{6}}{\left\|\phi_{23}\right\|_{M}^{2}}+\frac{\left|u_{0}\right|^{4}\left|v_{0}\right|^{8}}{\left\|\phi_{24}\right\|_{M}^{2}}+\frac{\left|u_{0}\right|^{6}}{\left\|\phi_{30}\right\|_{M}^{2}}+\frac{\left|u_{0}\right|^{6}\left|v_{0}\right|^{2}}{\left\|\phi_{31}\right\|_{M}^{2}}+\frac{\left|u_{0}\right|^{6}\left|v_{0}\right|^{4}}{\left\|\phi_{32}\right\|_{M}^{2}} \\
(3.1) \quad & +\frac{\left|u_{0}\right|^{6}\left|v_{0}\right|^{6}}{\left\|\phi_{33}\right\|_{M}^{2}}+\frac{\left|u_{0}\right|^{6}\left|v_{0}\right|^{8}}{\left\|\phi_{34}\right\|_{M}^{2}}+\frac{\left|u_{0}\right|^{6}\left|v_{0}\right|^{10}}{\left\|\phi_{35}\right\|_{M}^{2}}+\frac{\left|u_{0}\right|^{6}\left|v_{0}\right|^{12}}{\left\|\phi_{36}\right\|_{M}^{2}}+\cdots
\end{aligned}
$$

Theorem 3.3. Assume that $W_{0}$ is a complete Reinhardt domain. Then the Bergman function for the strongly pseudoconvex manifold $M$ is given by

$$
\begin{aligned}
B_{M}\left(\left(u_{0}, v_{0}\right),\left(\overline{u_{0}, v_{0}}\right)\right)= & \left\|\phi_{00}\right\|_{M}^{2} \Theta_{M}\left[1-\left\|\phi_{00}\right\|_{M}^{2} \Theta_{M}+\left(\left\|\phi_{00}\right\|_{M}^{2} \Theta_{M}\right)^{2}\right. \\
& \left.-\left(\left\|\phi_{00}\right\|_{M}^{2} \Theta_{M}\right)^{3}+\left(\left\|\phi_{00}\right\|_{M}^{2} \Theta_{M}\right)^{4}-\cdots\right] .
\end{aligned}
$$

The Bergman function for the strongly pseudoconvex variety $V$ is given by

$$
\begin{aligned}
B_{V}((x, y, z),(\overline{x, y, z}))= & \left\|\phi_{00}\right\|_{M}^{2} \Theta_{V}\left[1-\left\|\phi_{00}\right\|_{M}^{2} \Theta_{V}+\left(\left\|\phi_{00}\right\|_{M}^{2} \Theta_{V}\right)^{2}\right. \\
& \left.-\left(\left\|\phi_{00}\right\|_{M}^{2} \Theta_{V}\right)^{3}+\left(\left\|\phi_{00}\right\|_{M}^{2} \Theta_{V}\right)^{4}-\cdots\right]
\end{aligned}
$$

where

$$
\begin{aligned}
& \Theta_{V}=\frac{|x|^{2}}{\left\|\phi_{10}\right\|_{M}^{2}}+\frac{|z|^{2}}{\left\|\phi_{11}\right\|_{M}^{2}}+\frac{|y|^{2}}{\left\|\phi_{12}\right\|_{M}^{2}}+\frac{|x|^{4}}{\left\|\phi_{20}\right\|_{M}^{2}}+\frac{|x|^{2}|z|^{2}}{\left\|\phi_{21}\right\|_{M}^{2}}+\frac{|z|^{4}}{\left\|\phi_{22}\right\|_{M}^{2}} \\
& +\frac{|y|^{2}|z|^{2}}{\left\|\phi_{23}\right\|_{M}^{2}}+\frac{|y|^{4}}{\left\|\phi_{24}\right\|_{M}^{2}}+\frac{|x|^{6}}{\left\|\phi_{30}\right\|_{M}^{2}}+\frac{|x|^{5}|y|}{\left\|\phi_{31}\right\|_{M}^{2}}+\frac{|x|^{4}|y|^{2}}{\left\|\phi_{32}\right\|_{M}^{2}}+\frac{|z|^{6}}{\left\|\phi_{33}\right\|_{M}^{2}} \\
& +\frac{|x|^{2}|y|^{4}}{\left\|\phi_{34}\right\|_{M}^{2}}+\frac{|x||y|^{5}}{\left\|\phi_{35}\right\|_{M}^{2}}+\frac{|y|^{6}}{\left\|\phi_{36}\right\|_{M}^{2}}+\cdots
\end{aligned}
$$

Proof: $B_{M}\left(\left(u_{0}, v_{0}\right),\left(\overline{u_{0}, v_{0}}\right)\right)=\frac{K_{M, 0}}{K_{M}}=\frac{\Theta_{M}}{\frac{1}{\left\|\phi_{00}\right\|_{M}^{2}}+\Theta_{M}}=\frac{\left\|\phi_{00}\right\|_{M}^{2} \Theta_{M}}{1+\left\|\phi_{00}\right\|_{M}^{2} \Theta_{M}}$. 
Hence (3.2) follows immediately. Recall that the resolution map is given by $(x, y, z)=\left(u, u_{0} v_{0}^{2}, u_{0} v_{0}\right)$. Then (3.4) and (3.3) follow from (3.1) and (3.2) respectively.

Q.E.D.

Lemma 3.4. Any biholomorphism $\Psi=\left(\psi_{1}, \psi_{2}, \psi_{3}\right): V \rightarrow V$ has the following representation

$\left(\begin{array}{l}\psi_{1}(x, y, z) \\ \psi_{2}(x, y, z) \\ \psi_{3}(x, y, z)\end{array}\right)=\left(\begin{array}{lll}a_{11} & a_{12} & a_{13} \\ a_{21} & a_{22} & a_{23} \\ a_{31} & a_{32} & a_{33}\end{array}\right)\left(\begin{array}{l}x \\ y \\ z\end{array}\right)+$ higher order terms in $x, y$ and $z$

where the constants $a_{i j}$ satisfy the following equations

$$
\begin{aligned}
& a_{11} a_{21}-a_{31}^{2}=0 \\
& a_{12} a_{22}-a_{32}^{2}=0 \\
& a_{13} a_{23}-a_{33}^{2}+a_{11} a_{22}+a_{12} a_{21}-2 a_{31} a_{32}=0 \\
& a_{11} a_{23}+a_{13} a_{21}-2 a_{31} a_{33}=0 \\
& a_{12} a_{23}+a_{13} a_{22}-2 a_{32} a_{33}=0 \\
& \operatorname{det}\left(a_{i j}\right) \neq 0 .
\end{aligned}
$$

Proof. Since $\Psi: V \rightarrow V$, we have $\psi_{1}(x, y, z) \psi_{2}(x, y, z)-\psi_{3}^{2}(x, y, z)=0$. By looking at the quadratic part of this equation, we obtain

$$
\left(a_{11} x+a_{12} y+a_{13} z\right)\left(a_{21} x+a_{22} y+a_{23} z\right)-\left(a_{31} x+a_{32} y+a_{33} z\right)^{2}=0
$$

which implies

$$
\begin{aligned}
& \left(a_{11} a_{21}-a_{31}^{2}\right) x^{2}+\left(a_{11} a_{22}-a_{32}^{2}\right) y^{2}+\left(a_{13} a_{23}-a_{33}^{2}\right) z^{2} \\
& +\left(a_{11} a_{22}+a_{12} a_{21}-2 a_{31} a_{32}\right) x y+\left(a_{11} a_{23}+a_{13} a_{21}-2 a_{31} a_{33}\right) x z \\
& +\left(a_{12} a_{23}+a_{13} a_{22}-2 a_{32} a_{33}\right) y z=0 .
\end{aligned}
$$

Since $z^{2}=x y$, (3.5)-(3.9) follows from (3.11). (3.10) is a consequence of the fact that $\Psi$ is a biholomorphism.

Proposition 3.5. Let $V_{i}=\left\{(x, y, z) \in \mathbb{C}^{3}: x y=z^{2}\right.$ and $\eta_{i}(x, y, z)<\epsilon_{0}$, where $\eta_{i}$ is a strictly plurisubharmonic Reinhardt function $\}$ for $i=1,2$. Let $M_{i}=\widetilde{\pi}^{-1}\left(V_{i}\right), i=1,2$. Suppose that $\Psi: V_{1} \rightarrow V_{2}$ is a biholomorphic map given by $\Psi(x, y, z)=\left(a_{11} x+a_{12} y+a_{13} z, a_{21} x+a_{22} y+a_{23} z, a_{31} x+a_{32} y+a_{33} z\right)+$ 
higher order terms. Then

$$
\begin{aligned}
& \frac{\left\|\phi_{00}\right\|_{M_{2}}^{2}}{\left\|\phi_{10}\right\|_{M_{2}}^{2}}\left|a_{11}\right|^{2}+\frac{\left\|\phi_{00}\right\|_{M_{2}}^{2}}{\left\|\phi_{11}\right\|_{M_{2}}^{2}}\left|a_{31}\right|^{2}+\frac{\left\|\phi_{00}\right\|_{M_{2}}^{2}}{\left\|\phi_{12}\right\|_{M_{2}}^{2}}\left|a_{21}\right|^{2}=\frac{\left\|\phi_{00}\right\|_{M_{1}}^{2}}{\left\|\phi_{10}\right\|_{M_{1}}^{2}} \\
& \frac{\left\|\phi_{00}\right\|_{M_{2}}^{2}}{\left\|\phi_{10}\right\|_{M_{2}}^{2}}\left|a_{12}\right|^{2}+\frac{\left\|\phi_{00}\right\|_{M_{2}}^{2}}{\left\|\phi_{11}\right\|_{M_{2}}^{2}}\left|a_{32}\right|^{2}+\frac{\left\|\phi_{00}\right\|_{M_{2}}^{2}}{\left\|\phi_{12}\right\|_{M_{2}}^{2}}\left|a_{22}\right|^{2}=\frac{\left\|\phi_{00}\right\|_{M_{1}}^{2}}{\left\|\phi_{12}\right\|_{M_{1}}^{2}} \\
& \frac{\left\|\phi_{00}\right\|_{M_{2}}^{2}}{\left\|\phi_{10}\right\|_{M_{2}}^{2}}\left|a_{13}\right|^{2}+\frac{\left\|\phi_{00}\right\|_{M_{2}}^{2}}{\left\|\phi_{11}\right\|_{M_{2}}^{2}}\left|a_{33}\right|^{2}+\frac{\left\|\phi_{00}\right\|_{M_{2}}^{2}}{\left\|\phi_{12}\right\|_{M_{2}}^{2}}\left|a_{23}\right|^{2}=\frac{\left\|\phi_{00}\right\|_{M_{1}}^{2}}{\left\|\phi_{11}\right\|_{M_{1}}^{2}} \\
& \frac{\left\|\phi_{00}\right\|_{M_{2}}^{2}}{\left\|\phi_{10}\right\|_{M_{2}}^{2}} a_{11} \overline{a_{12}}+\frac{\left\|\phi_{00}\right\|_{M_{2}}^{2}}{\left\|\phi_{11}\right\|_{M_{2}}^{2}} a_{31} \overline{a_{32}}+\frac{\left\|\phi_{00}\right\|_{M_{2}}^{2}}{\left\|\phi_{12}\right\|_{M_{2}}^{2}} a_{21} \overline{a_{22}}=0 \\
& \frac{\left\|\phi_{00}\right\|_{M_{2}}^{2}}{\left\|\phi_{10}\right\|_{M_{2}}^{2}} a_{11} \overline{a_{13}}+\frac{\left\|\phi_{00}\right\|_{M_{2}}^{2}}{\left\|\phi_{11}\right\|_{M_{2}}^{2}} a_{31} \overline{a_{33}}+\frac{\left\|\phi_{00}\right\|_{M_{2}}^{2}}{\left\|\phi_{12}\right\|_{M_{2}}^{2}} a_{21} \overline{a_{23}}=0 \\
& \left\|\phi_{00}\right\|_{M_{2}}^{2} a_{12} \overline{a_{13}}+\frac{\left\|\phi_{00}\right\|_{M_{2}}^{2}}{\left\|\phi_{11}\right\|_{M_{2}}^{2}} a_{32} \overline{a_{33}}+\frac{\left\|\phi_{00}\right\|_{M_{2}}^{2}}{\left\|\phi_{12}\right\|_{M_{2}}^{2}} a_{22} \overline{a_{23}}=0 \\
& \frac{\left\|\phi_{10}\right\|_{M_{2}}^{2}}{\|}=0
\end{aligned}
$$

Proof. $B_{V_{1}}((x, y, z),(\overline{x, y, z}))=\left\|\phi_{00}\right\|_{M_{1}}^{2} \Theta_{V_{1}}-\left\|\phi_{00}\right\|_{M_{1}}^{4} \Theta_{V_{1}}^{2}+\left\|\phi_{00}\right\|_{M_{1}}^{6} \Theta_{V_{1}}^{3} \ldots$

$$
=\frac{\left\|\phi_{00}\right\|_{M_{1}}^{2}}{\left\|\phi_{10}\right\|_{M_{1}}^{2}}|x|^{2}+\frac{\left\|\phi_{00}\right\|_{M_{1}}^{2}|z|^{2}}{\left\|\phi_{11}\right\|_{M_{1}}^{3}}+\frac{\left\|\phi_{00}\right\|_{M_{1}}^{2}}{\left\|\Phi_{12}\right\|_{M_{1}}^{2}}|y|^{2}+\text { higher order term. }
$$

In view of Theorem 2.8 , we have

$$
\left.B_{V_{1}}((x, y, z)),(\overline{x, y, z})\right)=B_{V_{2}}(\Psi(x, y, z), \overline{\Psi(x, y, z)})
$$


which implies

$$
\begin{aligned}
& \frac{\left\|\phi_{00}\right\|_{M_{1}}^{2}|x|^{2}}{\left\|\phi_{10}\right\|_{M_{1}}^{2}}+\frac{\left\|\phi_{00}\right\|_{M_{1}}^{2}|z|^{2}}{\left\|\phi_{11}\right\|_{M_{1}}^{2}}+\frac{\left\|\phi_{00}\right\|_{M_{1}}^{2}|y|^{2}}{\left\|\phi_{12}\right\|_{M_{1}}^{2}} \\
& =\frac{\left\|\phi_{00}\right\|_{M_{2}}^{2}}{\left\|\phi_{10}\right\|_{M_{2}}^{2}}\left|a_{11} x+a_{12} y+a_{13} z\right|^{2}+\frac{\left\|\phi_{00}\right\|_{M_{2}}^{2}}{\left\|\phi_{11}\right\|_{M_{2}}^{2}}\left|a_{31} x+a_{32} y+a_{33} z\right|^{2} \\
& +\frac{\left\|\phi_{00}\right\|_{M_{2}}^{2}}{\left\|\phi_{12}\right\|_{M_{2}}^{2}}\left|a_{21} x+a_{22} y+a_{23} z\right|^{2} \\
& =\left(\frac{\left\|\phi_{00}\right\|_{M_{2}}^{2}}{\left\|\phi_{10}\right\|_{M_{2}}^{2}}\left|a_{11}\right|^{2}+\frac{\left\|\phi_{00}\right\|_{M_{2}}^{2}}{\left\|\phi_{11}\right\|_{M_{2}}^{2}}\left|a_{31}\right|^{2}+\frac{\left\|\phi_{00}\right\|_{M_{2}}^{2}}{\left\|\phi_{12}\right\|_{M_{2}}^{2}}\left|a_{21}\right|^{2}\right)|x|^{2} \\
& +\left(\frac{\left\|\phi_{00}\right\|_{M_{2}}^{2}}{\left\|\phi_{10}\right\|_{M_{2}}^{2}}\left|a_{12}\right|^{2}+\frac{\left\|\phi_{00}\right\|_{M_{2}}^{2}}{\left\|\phi_{11}\right\|_{M_{2}}^{2}}\left|a_{32}\right|^{2}+\frac{\left\|\phi_{00}\right\|_{M_{2}}^{2}}{\left\|\phi_{12}\right\|_{M_{2}}^{2}}\left|a_{22}\right|^{2}\right)|y|^{2} \\
& +\left(\frac{\left\|\phi_{00}\right\|_{M_{2}}^{2}}{\left\|\phi_{10}\right\|_{M_{2}}^{2}}\left|a_{13}\right|^{2}+\frac{\left\|\phi_{00}\right\|_{M_{2}}^{2}}{\left\|\phi_{11}\right\|_{M_{2}}^{2}}\left|a_{33}\right|^{2}+\frac{\left\|\phi_{00}\right\|_{M_{2}}^{2}}{\left\|\phi_{12}\right\|_{M_{2}}^{2}}\left|a_{23}\right|^{2}\right)|z|^{2} \\
& +\left(\frac{\left\|\phi_{00}\right\|_{M_{2}}^{2}}{\left\|\phi_{10}\right\|_{M_{2}}^{2}} a_{11} \overline{a_{12}}+\frac{\left\|\phi_{00}\right\|_{M_{2}}^{2}}{\left\|\phi_{11}\right\|_{M_{2}}^{2}} a_{31} \overline{a_{32}}+\frac{\left\|\phi_{00}\right\|_{M_{2}}^{2}}{\left\|\phi_{12}\right\|_{M_{2}}^{2}} a_{21} \overline{\overline{a_{22}}}\right) x \bar{y} \\
& +\left(\frac{\left\|\phi_{00}\right\|_{M_{2}}^{2}}{\left\|\phi_{10}\right\|_{M_{2}}^{2}} \overline{a_{11}} a_{12}+\frac{\left\|\phi_{00}\right\|_{M_{2}}^{2}}{\left\|\phi_{11}\right\|_{M_{2}}^{2}} \overline{a_{31}} a_{33}+\frac{\left\|\phi_{00}\right\|_{M_{2}}^{2}}{\left\|\phi_{12}\right\|_{M_{2}}^{2}} \overline{a_{21}} a_{22}\right) \bar{x} y \\
& +\left(\frac{\left\|\phi_{00}\right\|_{M_{2}}^{2}}{\left\|\phi_{10}\right\|_{M_{2}}^{2}} a_{11} \overline{a_{13}}+\frac{\left\|\phi_{00}\right\|_{M_{2}}^{2}}{\left\|\phi_{11}\right\|_{M_{2}}^{2}} a_{31} \overline{a_{33}}+\frac{\left\|\phi_{00}\right\|_{M_{2}}^{2}}{\left\|\phi_{12}\right\|_{M_{2}}^{2}} a_{21} \overline{a_{23}}\right) x \bar{z} \\
& +\left(\frac{\left\|\phi_{00}\right\|_{M_{2}}^{2}}{\left\|\phi_{10}\right\|_{M_{2}}^{2}} a_{11} \overline{a_{13}}+\frac{\left\|\phi_{00}\right\|_{M_{2}}^{2}}{\left\|\phi_{11}\right\|_{M_{2}}^{2}} \overline{a_{31}} a_{33}+\frac{\left\|\phi_{00}\right\|_{M_{2}}^{2}}{\left\|\phi_{12}\right\|_{M_{2}}^{2}} \overline{a_{21}} a_{23}\right) \bar{x} z \\
& +\left(\frac{\left\|\phi_{00}\right\|_{M_{2}}^{2}}{\left\|\phi_{10}\right\|_{M_{2}}^{2}} a_{12} \overline{a_{13}}+\frac{\left\|\phi_{00}\right\|_{M_{2}}^{2}}{\left\|\phi_{11}\right\|_{M_{2}}^{2}} a_{32} \overline{a_{33}}+\frac{\left\|\phi_{00}\right\|_{M_{2}}^{2}}{\left\|\phi_{12}\right\|_{M_{2}}^{2}} a_{22} \overline{a_{23}}\right) y \bar{z} \\
& +\left(\frac{\left\|\phi_{00}\right\|_{M_{2}}^{2}}{\left\|\phi_{10}\right\|_{M_{2}}^{2}} \overline{a_{12}} a_{13}+\frac{\left\|\phi_{00}\right\|_{M_{2}}^{2}}{\left\|\phi_{11}\right\|_{M_{2}}^{2}} \overline{a_{32}} a_{33}+\frac{\left\|\phi_{00}\right\|_{M_{2}}^{2}}{\left\|\phi_{12}\right\|_{M_{2}}^{2}} \overline{a_{22}} a_{23}\right) y \bar{z}
\end{aligned}
$$

(3.12)-(3.17) follows immediately.

The following theorem gives a continuous numerical invariant for strongly pseudoconvex CR manifolds lying in $\widetilde{V}=\left\{(x, y, z) \in \mathbb{C}^{3}: x y=z^{2}\right\}$.

Theorem 3.6. Let $V=\left\{(x, y, z) \in \mathbb{C}^{3}: x y=z^{2}\right.$ and $\eta(x, y, z)<\epsilon_{0}$ where $\eta$ is a strictly plurisubharmonic Reinhardt function $\}$ such that $X=\partial V$ is a smooth CR manifold. Let $M=\widetilde{\pi}^{-1}(V)$. With the notation in Proposition 3.2, $\nu_{X}:=\frac{\left\|\phi_{11}\right\|_{M}^{2}}{\left\|\phi_{10}\right\|_{M}\left\|\phi_{12}\right\|_{M}}$ is a $C R$ invariant of $X$ in $V$, i.e. if $X_{1}$ and $X_{2}$ are two such strongly pseudoconvex $C R$ manifolds in $V$ which are $C R$ equivalent, then 
$\frac{\left\|\Phi_{11}\right\|_{M_{1}}^{2}}{\left\|\phi_{10}\right\|_{M_{1}}\left\|\phi_{12}\right\|_{M_{1}}}=\frac{\left\|\phi_{11}\right\|_{M_{2}}^{2}}{\left\|\phi_{10}\right\|_{M_{2}}\left\|\phi_{12}\right\|_{M_{2}}}$, where $M_{1}$ and $M_{2}$ are strongly pseudoconvex CR manifolds which have $X_{1}$ and $X_{2}$ as boundaries respectively.

Proof. Let $V_{i}=\left\{(x, y, z) \in \mathbb{C}^{3}: x y=z^{2}\right.$ and $\eta_{i}(x, y, z)<\epsilon_{0}$, where $\eta_{i}$ is a strictly plurisubharmonic Reinhardt function $\}$ and $\partial V_{i}=X_{i}, i=1,2$. If $X_{i}$ is CR equivalent to $X_{2}$, then $V_{1}$ is biholomorphic equivalent to $V_{2}$ by Theorem 3.1. Theorem 3.6 follows from the following Theorem 3.7.

Theorem 3.7. Let $V_{i}=\left\{(x, y, z) \in \mathbb{C}^{3}: x y=z^{2}\right.$ and $\eta_{i}(x, y, z)<\epsilon_{0}$, where $\eta_{i}$ is a strictly plurisubharmonic Reinhardt function $\}$ and $M_{i}=\widetilde{\pi}^{-1}\left(V_{i}\right)$ is the resolution of singularity of $V_{i}, i=1,2$. If there exists a biholomorphic map $\Psi$ from $V_{1}$ to $V_{2}$ and $\frac{\left\|\phi_{11}\right\|_{M_{2}}^{2}}{\left\|\phi_{10}\right\|_{M_{2}}\left\|\phi_{12}\right\|_{M_{2}}} \neq \frac{1}{2}$, then $\frac{\left\|\phi_{11}\right\|_{M_{1}}^{2}}{\left\|\phi_{10}\right\|_{M_{1}}\left\|\phi_{12}\right\|_{M_{1}}}=\frac{\left\|\phi_{11}\right\|_{M_{2}}^{2}}{\left\|\phi_{10}\right\|_{M_{2}}\left\|\phi_{12}\right\|_{M_{2}}}$.

Proof. The same argument as in Lemma 3.4 will show that $\Psi$ can be written as $\left(\psi_{1}, \psi_{2}, \psi_{3}\right)=\left(a_{11} x+a_{12} y+a_{13} z, a_{21} x+a_{22} y+a_{23} z, a_{31} x+a_{32} y+a_{33} z\right)+$ higher order terms such that (3.5)-(3.10) hold. By Proposition 3.5, we know that (3.12)-(3.17) also hold. We have three cases to consider: Case 1, $a_{31} \neq 0$ and $a_{32} \neq 0$; Case 2, $a_{31}=0$; Case $3, a_{32}=0$.

Case 1: $a_{31} \neq 0$ and $a_{32} \neq 0$. In view of (3.5) and (3.6), we have $a_{11} \neq 0, a_{21} \neq$ $0, a_{12} \neq 0$ and $a_{22} \neq 0$ in this case.

$$
\begin{aligned}
(3.5) & \Longrightarrow \frac{a_{11}}{a_{31}}=\frac{a_{31}}{a_{21}}:=r_{1} \neq 0 \\
& \Longrightarrow a_{11}=r_{1} a_{31}, \quad a_{21}=\frac{1}{r_{1}} a_{31} \\
(3.6) & \Longrightarrow \frac{a_{22}}{a_{32}}=\frac{a_{32}}{a_{12}}:=r_{2} \neq 0 \\
& \Longrightarrow a_{22}=r_{2} a_{32}, \quad a_{12}=\frac{1}{r_{2}} a_{32} \\
(3.8) \text { and }(3.18) & \Longrightarrow r_{1} a_{23}+\frac{1}{r_{1}} a_{13}-2 a_{33}=0 \\
(3.9) \text { and }(3.19) & \Longrightarrow \frac{1}{r_{2}} a_{23}+r_{2} a_{13}-2 a_{33}=0 \\
(3.20) \text { and }(3.21) & \Longrightarrow\left(r_{1}-\frac{1}{r_{2}}\right) a_{23}+\left(\frac{1}{r_{1}}-r_{2}\right) a_{13}=0 .
\end{aligned}
$$$$
\text { (3.9) and }(3.19) \Longrightarrow \frac{1}{r_{2}} a_{23}+r_{2} a_{13}-2 a_{33}=0
$$

There are two cases to be considered. 
Case $1(\mathbf{a}): r_{1}-\frac{1}{r_{2}}=0$, i.e. $r_{2}=\frac{1}{r_{1}}$

$$
\begin{aligned}
(3.19) & \Longrightarrow a_{22}=\frac{1}{r_{1}} a_{32}, \quad a_{12}=r_{1} a_{32} \\
(3.20) & \Longrightarrow a_{33}=\frac{1}{2} r_{1} a_{23}+\frac{1}{2 r_{1}} a_{13} \\
(3.7),(3.18) \text { and }(3.23) & \Longrightarrow a_{13} a_{23}-a_{33}^{2}=0 \\
(3.25) \text { and }(3.24) & \Longrightarrow a_{13}=r_{1}^{2} a_{23} \\
(3.24) \text { and }(3.26) & \Longrightarrow a_{33}=r_{1} a_{23}=\frac{1}{r_{1}} a_{13}
\end{aligned}
$$$$
\text { (3.25) and }(3.24) \Longrightarrow a_{13}=r_{1}^{2} a_{23}
$$

(3.15), (3.18) and (3.23) imply

$$
\frac{\left\|\phi_{00}\right\|_{M_{2}}^{2}}{\left\|\phi_{10}\right\|_{M_{2}}^{2}}\left|r_{1}\right|^{2}+\frac{\left\|\phi_{00}\right\|_{M_{2}}^{2}}{\left\|\phi_{11}\right\|_{M_{2}}^{2}}+\frac{\left\|\phi_{00}\right\|_{M_{2}}^{2}}{\left\|\phi_{12}\right\|_{M_{2}}^{2}} \frac{1}{\left|r_{1}\right|^{2}}=0
$$

which is a contradiction because the left hand side of (3.28) is positive. Hence Case 1 (a) cannot happen.

Case 1 (b): $r_{1}-\frac{1}{r_{2}} \neq 0$

$$
\begin{aligned}
(3.22) & \Longrightarrow a_{23}=\frac{r_{2}}{r_{1}} a_{13} \\
(3.20) \text { and }(3.29) & \Longrightarrow a_{33}=\left(\frac{r_{2}}{2}+\frac{1}{2 r_{1}}\right) a_{13} .
\end{aligned}
$$

In view of (3.29) and (3.30), we have $a_{13} \neq 0$ because $\operatorname{det}\left(a_{i j}\right) \neq 0$. (3.15), (3.18) and (3.19) imply

$$
\frac{\left\|\phi_{00}\right\|_{M_{2}}^{2}}{\left\|\phi_{10}\right\|_{M_{2}}^{2}} \frac{r_{1}}{\overline{r_{2}}}+\frac{\left\|\phi_{00}\right\|_{M_{2}}^{2}}{\left\|\phi_{11}\right\|_{M_{2}}^{2}}+\frac{\left\|\phi_{00}\right\|_{M_{2}}^{2}}{\left\|\phi_{12}\right\|_{M_{2}}^{2}} \frac{\overline{r_{2}}}{r_{1}}=0
$$

(3.16) and (3.18), (3.29) and (3.30) imply

$$
\frac{\left\|\phi_{00}\right\|_{M_{2}}^{2}}{\left\|\phi_{10}\right\|_{M_{2}}^{2}} r_{1}+\frac{\left\|\phi_{00}\right\|_{M_{2}}^{2}}{\left\|\phi_{11}\right\|_{M_{2}}^{2}}\left(\frac{\overline{r_{2}}}{2}+\frac{1}{2 \overline{r_{1}}}\right)+\frac{\left\|\phi_{00}\right\|_{M_{2}}^{2}}{\left\|\phi_{12}\right\|_{M_{2}}^{2}} \frac{\overline{r_{2}}}{\left|r_{1}\right|^{2}}=0
$$

(3.17), (3.19), (3.29) and (3.30) imply

$$
\frac{\left\|\phi_{00}\right\|_{M_{2}}^{2}}{\left\|\phi_{10}\right\|_{M_{2}}^{2}} \frac{1}{r_{2}}+\frac{\left\|\phi_{00}\right\|_{M_{2}}^{2}}{\left\|\phi_{11}\right\|_{M_{2}}^{2}}\left(\frac{\overline{r_{2}}}{2}+\frac{1}{2 \overline{r_{1}}}\right)+\frac{\left\|\phi_{00}\right\|_{M_{2}}^{2}}{\left\|\phi_{12}\right\|_{M_{2}}^{2}} \frac{\left|r_{2}\right|^{2}}{\overline{r_{1}}}=0
$$


Clearly (3.32), (3.33) and (3.34) imply

$$
\begin{gathered}
\operatorname{det}\left(\begin{array}{ccc}
\frac{r_{1}}{\bar{r}_{2}} & 1 & \frac{\overline{r_{2}}}{r_{1}} \\
r_{1} & \overline{\frac{r_{2}}{2}}+\frac{1}{2 \overline{r_{1}}} & \frac{\overline{r_{2}}}{\left|r_{1}\right|^{2}} \\
\frac{1}{r_{2}} & \overline{\frac{r_{2}}{2}}+\frac{1}{2 \bar{r}_{1}} & \frac{\left|r_{2}\right|^{2}}{\overline{r_{1}}}
\end{array}\right)=0 \\
\Longrightarrow\left(\overline{r_{1} r_{2}}+1\right)\left(r_{1} r_{2}+\overline{r_{2} r_{2}}\right)\left(r_{1} r_{2}-1\right)+2 \overline{r_{1} r_{2}}\left(1-r_{1} r_{2}\right)\left(1+r_{1} r_{2}\right)=0 .
\end{gathered}
$$

Since $r_{1}-\frac{1}{r_{2}} \neq 0$, i.e. $r_{1} r_{2}-1 \neq 0$, we have

$$
\Longrightarrow \quad \begin{aligned}
& \left(\overline{r_{1}} \overline{r_{2}}+1\right)\left(r_{1} r_{2}+\overline{r_{1} r_{2}}\right)-2 \overline{r_{1} r_{2}}\left(1+r_{1} r_{2}\right)=0 \\
& \left(r_{1} r_{2}-\overline{r_{1} r_{2}}\right)\left(1-\overline{r_{1} r_{2}}\right)=0 .
\end{aligned}
$$

Since $\overline{r_{1} r_{2}}-1 \neq 0$, we have

$$
r_{1} r_{2}=\overline{r_{1} r_{2}}
$$

Let $\alpha=\frac{r_{1}}{\bar{r}_{2}}$. Then $\alpha=\bar{\alpha}, r_{1}=\alpha \overline{r_{2}}, \overline{r_{1}}=\alpha r_{2}$. (3.31), (3.32) and (3.33) can be rewritten as

$$
\begin{aligned}
\alpha^{2}+\frac{\left\|\phi_{10}\right\|_{M_{2}}^{2}}{\left\|\phi_{11}\right\|_{M_{2}}^{2}} \alpha+\frac{\left\|\phi_{10}\right\|_{M_{2}}^{2}}{\left\|\phi_{12}\right\|_{M_{2}}^{2}} & =0 \\
(3.36) \quad \frac{\alpha^{3}\left|r_{2}\right|^{2}}{\left\|\phi_{10}\right\|_{M_{2}}^{2}}+\frac{1}{\left\|\phi_{11}\right\|_{M_{2}}^{2}}\left(\frac{\alpha^{2}\left|r_{2}\right|^{2}}{2}+\frac{\alpha}{2}\right)+\frac{1}{\left\|\phi_{12}\right\|_{M_{2}}^{2}} & =0 \\
(3.37) \quad \frac{\alpha}{\left\|\phi_{10}\right\|_{M_{2}}^{2}}+\frac{1}{\left\|\phi_{11}\right\|_{M_{2}}^{2}}\left(\frac{\alpha\left|r_{2}\right|^{2}}{2}+\frac{1}{2}\right)+\frac{\left|r_{2}\right|^{2}}{\left\|\phi_{12}\right\|_{M_{2}}^{2}} & =0 \\
(3.36)-\alpha(3.37) \Rightarrow & \frac{\alpha^{2}\left(\alpha\left|r_{2}\right|^{2}-1\right)}{\left\|\phi_{10}\right\|_{M_{2}}^{2}}+\frac{1-\alpha\left|r_{2}\right|^{2}}{\left\|\phi_{12}\right\|_{M_{2}}^{2}}=0
\end{aligned}
$$$$
\text { i.e. } 1-\alpha\left|r_{2}\right|^{2}=0 \quad \text { or } \quad \alpha^{2}=\frac{\left\|\phi_{10}\right\|_{M_{2}}^{2}}{\left\|\phi_{12}\right\|_{M_{2}}^{2}} \text {. }
$$

If $\alpha^{2}=\frac{\left\|\phi_{10}\right\|_{M_{2}}^{2}}{\left\|\phi_{12}\right\|_{M_{2}}^{2}}$, then (3.35) implies $\alpha=\frac{-2\left\|\phi_{11}\right\|_{M_{2}}^{2}}{\left\|\phi_{12}\right\|_{M_{2}}^{2}}$. It follows easily that $\frac{\left\|\phi_{11}\right\|_{M_{2}}^{2}}{\left\|\phi_{12}\right\|_{M_{2}}\left\|\phi_{10}\right\|_{M_{2}}}=\frac{1}{2}$, a contradiction to our hypothesis. Hence we conclude that

$$
\alpha\left|r_{2}\right|^{2}=1
$$

Putting (3.39) in (3.37), we get

$$
\frac{1}{\left|r_{2}\right|^{2}\left\|\phi_{10}\right\|_{M_{2}}^{2}}+\frac{1}{\left\|\phi_{11}\right\|_{M_{2}}^{2}}+\frac{\left|r_{2}\right|^{2}}{\left\|\phi_{12}\right\|_{M_{2}}^{2}}=0
$$


which is absurd since the left hand side is positive. Thus Case 1 (b) cannot occur also.

Case 2: $a_{31}=0$. By (3.5), we have either $a_{11}=0$ or $a_{21}=0$.

Case 2 (a): $a_{31}=0$ and $a_{11}=0$. Since $\operatorname{det}\left(a_{i j}\right) \neq 0$, we have $a_{21} \neq 0$.

$$
\begin{aligned}
(3.15) & \Longrightarrow a_{21} \overline{a_{22}}=0 \Longrightarrow a_{22}=0 \\
(3.8) & \Longrightarrow a_{13} a_{21}=0 \Longrightarrow a_{13}=0 \\
\text { (3.6) and }(3.40) & \Longrightarrow a_{32}=0 .
\end{aligned}
$$

Since $\operatorname{det}\left(a_{i j}\right) \neq 0$ and $a_{11}=0=a_{13}$, we have $a_{12} \neq 0$.

$$
\begin{aligned}
& \text { (3.9), (3.40) and (3.42) } \Longrightarrow a_{12} a_{23}=0 \Longrightarrow a_{23}=0 \\
& \text { (3.7) and (3.43) } \Longrightarrow-a_{33}^{2}+a_{12} a_{21}=0
\end{aligned}
$$$$
(3.12) \Longrightarrow\left|a_{21}\right|^{2}=\frac{\left\|\phi_{12}\right\|_{M_{2}}^{2}}{\left\|\phi_{00}\right\|_{M_{2}}^{2}} \cdot \frac{\left\|\phi_{00}\right\|_{M_{1}}^{2}}{\left\|\phi_{10}\right\|_{M_{1}}^{2}}
$$$$
(3.13) \Longrightarrow\left|a_{12}\right|^{2}=\frac{\left\|\phi_{10}\right\|_{M_{2}}^{2}}{\left\|\phi_{00}\right\|_{M_{2}}^{2}} \cdot \frac{\left\|\phi_{00}\right\|_{M_{1}}^{2}}{\left\|\phi_{12}\right\|_{M_{1}}^{2}}
$$$$
(3.14) \Longrightarrow\left|a_{33}\right|^{2}=\frac{\left\|\phi_{11}\right\|_{M_{2}}^{2}}{\left\|\phi_{00}\right\|_{M_{2}}^{2}} \cdot \frac{\left\|\phi_{00}\right\|_{M_{1}}^{2}}{\left\|\phi_{11}\right\|_{M_{1}}^{2}}
$$

(3.44), (3.45), (3.46) and (3.47) imply

$$
\frac{\left\|\phi_{11}\right\|_{M_{2}}^{2}}{\left\|\phi_{12}\right\|_{M_{2}}\left\|\phi_{10}\right\|_{M_{2}}}=\frac{\left\|\phi_{11}\right\|_{M_{1}}^{2}}{\left\|\phi_{10}\right\|_{M_{1}}\left\|\phi_{12}\right\|_{M_{1}}}
$$

Case 2 (b): $a_{31}=0$ and $a_{21}=0$. Since $\operatorname{det}\left(a_{i j}\right) \neq 0$, we have $a_{11} \neq 0$.

$$
(3.15) \quad \Longrightarrow \quad a_{11} \overline{a_{12}}=0 \Longrightarrow a_{12}=0
$$$$
(3.8) \Longrightarrow a_{11} a_{23}=0 \Longrightarrow a_{23}=0
$$

(3.6) and (3.48) $\Longrightarrow a_{32}=0$. 
Since $a_{32}=0=a_{12}$ and $\operatorname{det}\left(a_{i j}\right) \neq 0$, we have $a_{22} \neq 0$.

$$
\begin{aligned}
(3.9),(3.51) \text { and }(3.49) & \Longrightarrow a_{13} a_{22}=0 \Longrightarrow a_{13}=0 \\
(3.7),(3.52) \text { and }(3.49) & \Longrightarrow-a_{33}^{2}+a_{11} a_{22}=0 \\
(3.12) & \Longrightarrow\left|a_{11}\right|^{2}=\frac{\left\|\phi_{10}\right\|_{M_{2}}^{2}}{\left\|\phi_{00}\right\|_{M_{2}}^{2}} \cdot \frac{\left\|\phi_{00}\right\|_{M_{1}}^{2}}{\left\|\phi_{10}\right\|_{M_{1}}^{2}} \\
(3.13) & \Longrightarrow\left|a_{22}\right|^{2}=\frac{\left\|\phi_{12}\right\|_{M_{2}}^{2}}{\left\|\phi_{00}\right\|_{M_{2}}^{2}} \cdot \frac{\left\|\phi_{00}\right\|_{M_{1}}^{2}}{\left\|\phi_{12}\right\|_{M_{1}}^{2}} \\
(3.14) & \Longrightarrow\left|a_{33}\right|^{2}=\frac{\left\|\phi_{11}\right\|_{M_{2}}^{2}}{\left\|\phi_{00}\right\|_{M_{2}}^{2}} \cdot \frac{\left\|\phi_{00}\right\|_{M_{1}}^{2}}{\left\|\phi_{11}\right\|_{M_{1}}^{2}}
\end{aligned}
$$

(3.53), (3.54), (3.55) and (3.56) imply (3.48).

Case 3: $a_{32}=0$. By (3.6), we have either $a_{12}=0$ or $a_{22}=0$.

Case 3 (a): $a_{32}=0$ and $a_{12}=0$. By the same argument as above, we can show that all $a_{i j}$ are zero except $a_{11}, a_{22}$ and $a_{33}$. Moreover $a_{11}, a_{22}, a_{33}$ satisfy (3.53), (3.54), (3.55) and (3.56) so that (3.48) holds.

Case 3 (b): $a_{32}=0$ and $a_{22}=0$. By the same argument as above, we can show that all $a_{i j}$ are zero except $a_{12}, a_{21}$ and $a_{33}$. Moreover, $a_{12}, a_{21}, a_{33}$ satisfy (3.44), (3.45), (3.46) and (3.47) so that (3.48) holds.

Corollary 3.8. Let $V_{i}=\left\{(x, y, z) \in \mathbb{C}^{3}: x y=z^{2}\right.$ and $\eta_{i}(x, y, z)<\epsilon_{0}$, where $\eta_{i}$ is a strictly plurisubharmonic Reinhardt function $\}$. If the CR invariant $\nu_{X_{2}}$ in Theorem 3.6 is not equal to 0.5 , then the biholomorphic map $\Psi=\left(\psi_{1}, \psi_{2}, \psi_{3}\right)$ : $V_{1} \rightarrow V_{2}$ must be one of the following forms:

(1) $\left(\psi_{1}, \psi_{2}, \psi_{3}\right)=\left(a_{11} x, a_{22} y, a_{33} z\right)+$ higher order terms and $a_{33}^{2}=a_{11} a_{22}$.

(2) $\left(\psi_{1}, \psi_{2}, \psi_{3}\right)=\left(a_{12} y, a_{21} x, a_{33} z\right)+$ higher order terms and $a_{33}^{2}=a_{12} a_{21}$.

Proof: It is clear from the proof of Theorem 3.7.

\section{Explicit computation of new CR invariant}

Let $a$ be positive real number. We shall follow the notations in our previous section. Let $V_{a}=\left\{(x, y, z) \in \mathbb{C}^{3}: x y=z^{2}, a|x|^{2}+|y|^{2}+|z|^{2}<\epsilon_{0}\right\}$. Recall that $(x, y, z)=\left(u_{0}, u_{0} v_{0}^{2}, u_{0} v_{0}\right)$. Then $M_{a}$ be the resolution of $V_{a}$ with coordinate chart $W_{0}=\left\{\left(u_{0}, v_{0}\right): a\left|u_{0}\right|^{2}+\left|u_{0}\right|^{2}\left|v_{0}\right|^{4}+\left|u_{0}\right|^{2}\left|v_{0}\right|^{2}<\epsilon_{0}\right\}$. Next write $u_{0}=r e^{i \theta}$ 
and $v_{0}=\rho e^{i \phi}$. Then

$$
\begin{aligned}
\left\|\phi_{\alpha \beta}\right\|_{M_{a}}^{2} & =\int_{M_{a}} \phi_{\alpha \beta} \wedge \overline{\phi_{\alpha \beta}}=\int_{W_{0}}\left|u_{0}^{\alpha}\right|^{2}\left|v_{0}^{\beta}\right|^{2} d u_{0} \wedge d v_{0} \wedge d \overline{u_{0}} \wedge d \overline{v_{0}} \\
& =2 \pi \int_{0}^{2 \pi} \iint_{D} r^{2 \alpha+1} \rho^{2 \beta+1} d r d \rho d \theta
\end{aligned}
$$

where $D=\left\{(r, \rho): r \geq 0, \rho \geq 0, a r^{2}+r^{2} \rho^{4}+r^{2} \rho^{2}<\epsilon_{0}\right\}$. In particular

$$
\begin{aligned}
\left\|\phi_{1 \beta}\right\|_{M_{a}}^{2} & =2 \pi \int_{0}^{2 \pi} \int_{0}^{\infty} \int_{0}^{\frac{\sqrt{\epsilon_{0}}}{\sqrt{a+\rho^{2}+\rho^{4}}}} r^{3} \rho^{2 \beta+1} d r d \rho d \theta \\
& =2 \pi \int_{0}^{2 \pi} \int_{0}^{\infty} \frac{\epsilon_{0}^{2} \rho^{2 \beta+1}}{\left(a+\rho^{2}+\rho^{4}\right)^{2}} d \rho d \theta
\end{aligned}
$$

Therefore the new CR invariant for the CR manifold $X_{a}:=\partial V_{a}$ is

$$
\begin{aligned}
\nu_{a} & :=\frac{\left\|\phi_{11}\right\|_{M_{a}}^{2}}{\left\|\phi_{10}\right\|_{M_{a}}\left\|\phi_{12}\right\|_{M_{a}}} \\
& =\frac{\int_{0}^{2 \pi} \int_{0}^{\infty} \frac{\rho^{3}}{\left(a+\rho^{2}+\rho^{4}\right)^{2}} d \rho d \theta}{\left(\int_{0}^{2 \pi} \int_{0}^{\infty} \frac{\rho}{\left(a+\rho^{2}+\rho^{4}\right)^{2}} d \rho d \theta\right)^{\frac{1}{2}}\left(\int_{0}^{2 \pi} \int_{0}^{\infty} \frac{\rho^{5}}{\left(a+\rho^{2}+\rho^{4}\right)^{2}} d \rho d \theta\right)^{\frac{1}{2}}} .
\end{aligned}
$$

Case 1: $a=\frac{1}{4}$

$$
\begin{aligned}
I_{1} & =\int_{0}^{2 \pi} \int_{0}^{\infty} \frac{\rho}{\left(\rho^{4}+\rho^{2}+\frac{1}{4}\right)^{2}} d \rho d \theta=\pi \int_{0}^{\infty} \frac{1}{\left(x+\frac{1}{2}\right)^{4}} d x=\frac{8}{3} \pi \\
I_{2} & =\int_{0}^{2 \pi} \int_{0}^{\infty} \frac{\rho^{3}}{\left(\rho^{4}+\rho^{2}+\frac{1}{4}\right)^{2}} d \rho d \theta=\pi \int_{0}^{\infty} \frac{x}{\left(x+\frac{1}{2}\right)^{4}} d x=\frac{2}{3} \pi \\
I_{3} & =\int_{0}^{2 \pi} \int_{0}^{\infty} \frac{\rho^{5}}{\left(\rho^{4}+\rho^{2}+\frac{1}{4}\right)^{2}} d \rho d \theta=\pi \int_{0}^{\infty} \frac{x^{2}}{\left(x+\frac{1}{2}\right)^{4}} d x=\frac{2}{3} \pi \\
\nu_{\frac{1}{4}} & =\frac{I_{2}}{\sqrt{I_{1} I_{3}}}=\frac{\frac{2}{3} \pi}{\sqrt{\frac{8}{3} \pi \cdot \frac{2}{3} \pi}}=\frac{1}{2} .
\end{aligned}
$$


Case 2: $a>\frac{1}{4}$

$$
\begin{aligned}
I_{1} & =\int_{0}^{2 \pi} \int_{0}^{\infty} \frac{\rho}{\left(\rho^{4}+\rho^{2}+a\right)^{2}} d \rho d \theta=\pi \int_{0}^{\infty} \frac{d x}{\left(x^{2}+x+a\right)^{2}} \\
& =\left.\pi\left[\frac{2 x+1}{(4 a-1)\left(x^{2}+x+a\right)}+\frac{4}{(4 a-1)^{\frac{3}{2}}} \arctan \left(\frac{x+1}{\sqrt{4 a-1}}\right)\right]\right|_{0} ^{\infty} \\
& =\pi\left[\frac{\pi}{(4 a-1)^{\frac{3}{2}}}-\frac{1}{a(4 a-1)}-\frac{4}{(4 a-1)^{\frac{3}{2}}} \arctan \left(\frac{1}{\sqrt{4 a-1}}\right)\right] \\
I_{2} & =\int_{0}^{2 \pi} \int_{0}^{\infty} \frac{\rho^{3}}{\left(\rho^{4}+\rho^{2}+a\right)^{2}} d \rho d \theta=\pi \int_{0}^{\infty} \frac{x d x}{\left(x^{2}+x+a\right)^{2}} \\
= & \left.\pi\left[-\frac{2 a+x}{(4 a-1)\left(x^{2}+x+a\right)}-\frac{2}{(4 a-1)^{\frac{3}{2}}} \arctan \left(\frac{2 x+1}{\sqrt{4 a-1}}\right)\right]\right|_{0} ^{\infty} \\
I_{3} & =\pi\left[\frac{2}{4 a-1}-\frac{\pi}{(4 a-1)^{\frac{3}{2}}}+\frac{2}{(4 a-1)^{\frac{3}{2}}} \arctan \left(\frac{1}{\sqrt{4 a-1}}\right)\right] \\
& =\left.\pi\left[\frac{\rho^{5}}{(4 a-1)\left(x^{2}+x+a\right)}+\frac{4 a}{(4 a-1)^{\frac{3}{2}}} \arctan \left(\frac{2 x+1}{\sqrt{4 a-1}}\right)\right]\right|_{0} ^{\infty} \\
& =\pi\left[\frac{2 a \pi}{(4 a-1)^{\frac{3}{2}}-\frac{1}{4 a-1}}-\frac{4 a}{(4 a-1)^{\frac{3}{2}}} \arctan \left(\frac{1}{\sqrt{4 a-1}}\right)\right] .
\end{aligned}
$$

$$
\begin{aligned}
& \text { If } a=\frac{1}{2}, \text { then } I_{1}=\pi(\pi-2), I_{2}=\pi\left(2-\frac{\pi}{2}\right) \text { and } I_{3}=\pi\left(\frac{\pi}{2}-1\right) . \text { We have } \\
& \nu_{\frac{1}{2}}=\frac{2-\frac{\pi}{2}}{\sqrt{(\pi-2)\left(\frac{\pi}{2}-1\right)}}=\frac{\sqrt{2}(4-\pi)}{2(\pi-2)} \simeq 0.5317007373>0.5 .
\end{aligned}
$$

Similarly if $a=1>\frac{1}{4}$, then $I_{1}=\frac{\pi}{6}\left(\frac{4 \pi}{3 \sqrt{3}}-1\right), I_{2}=\frac{\pi}{3}\left(1-\frac{\pi}{3 \sqrt{3}}\right), I_{3}=I_{1}$ and $\nu_{1} \simeq 0.55753024284785>0.5$. 
Case 3: $a<\frac{1}{4}$

$$
\begin{aligned}
I_{1} & =\int_{0}^{2 \pi} \int_{0}^{\infty} \frac{\rho}{\left(\rho^{4}+\rho^{2}+a\right)} d \rho d \theta=\pi \int_{0}^{\infty} \frac{d x}{\left(x^{2}+x+a\right)^{2}} \\
& =\pi\left[\frac{1}{a(1-4 a)}+\frac{2}{(1-4 a)^{\frac{3}{2}}} \ln \frac{1-\sqrt{1-4 a}}{1+\sqrt{1-4 a}}\right] \\
I_{2} & =\int_{0}^{2 \pi} \int_{0}^{\infty} \frac{\rho^{3}}{\left(\rho^{4}+\rho^{2}+a\right)^{2}} d \rho d \theta=\pi \int_{0}^{\infty} \frac{x}{\left(x^{2}+x+a\right)^{2}} d x \\
& =\pi\left[-\frac{2}{1-4 a}-\frac{1}{(1-4 a)^{\frac{3}{2}}} \ln \frac{1-\sqrt{1-4 a}}{1+\sqrt{1-4 a}}\right] \\
I_{3} & =\int_{0}^{2 \pi} \int_{0}^{\infty} \frac{\rho^{5}}{\left(\rho^{4}+\rho^{2}+a\right)^{2}} d \rho d \theta=\pi \int_{0}^{\infty} \frac{x^{2}}{\left(x^{2}+x+a\right)^{2}} d x \\
& =\frac{\pi}{2}\left[\frac{1}{1-4 a}+\frac{2 a}{(1-4 a)^{\frac{3}{2}}} \ln \frac{1-\sqrt{1-4 a}}{1+\sqrt{1-4 a}}\right] .
\end{aligned}
$$

If $a=\frac{3}{16}<\frac{1}{4}$, then $I_{1}=\pi\left(\frac{64}{3}-16 \ln 3\right), I_{2}=\pi(8 \ln 3-8)$ and $I_{3}=$ $\pi(4-3 \ln 3)$. Hence

$$
\begin{aligned}
\nu_{\frac{3}{16}} & =\frac{8 \ln 3-8}{\sqrt{\left(\frac{64}{3}-16 \ln 3\right)(4-3 \ln 3)}}=\frac{2 \sqrt{3}(\ln 3-1)}{4-3 \ln 3} \\
& \simeq 0.4851191037356<0.5 .
\end{aligned}
$$

Similarly one has the following results:

$$
\begin{array}{ll}
a=\frac{2}{9}<\frac{1}{4}, & \nu_{\frac{2}{9}} \simeq 0.4940697511735 \\
a=\frac{15}{64}<\frac{1}{4}, & \nu_{\frac{15}{64}} \simeq 0.49674784168264 \\
a=\frac{6}{25}<\frac{1}{4}, & \nu_{\frac{6}{25}} \simeq 0.49794879674687 .
\end{array}
$$

\section{Automorphism group of compact CR manifold}

In this section, we shall show that our Bergman function can be used to determine the automorphism group of a compact CR manifold.

Theorem 5.1. Let $X=\left\{(x, y, z) \in \mathbb{C}^{3}: x y=z^{2}, \eta(x, y . z)<\epsilon_{0}\right\}$ where $\eta$ is a strictly plurisubharmonic Reinhardt function and $\nu_{X}$ be the CR invariant defined in the previous section, where $X=\partial V$. Then the automorphism group of $X$ for $\nu_{X} \neq \frac{1}{2}$ consists of biholomorphic map $\Psi=\left(\psi_{1}, \psi_{2}, \psi_{3}\right)$ of the following forms:

(1) $\left(\psi_{1}, \psi_{2}, \psi_{3}\right)=\left(e^{i \theta_{1}} x, e^{i \theta_{2}} y, e^{i \frac{\theta_{1}+\theta_{2}}{2}} z\right)$

(2) $\left(\psi_{1}, \psi_{2}, \psi_{3}\right)=\left(e^{i \theta_{1}} y, e^{i \theta_{2}} x, e^{i \frac{\theta_{1}+\theta_{2}}{2}} z\right)$. 
Proof. In view of Corollary 3.8, we know that $\Psi=\left(\psi_{1}, \psi_{2}, \psi_{3}\right)$ must be one of the following forms:

(1) $\left(\psi_{1}, \psi_{2}, \psi_{3}\right)=\left(a_{11} x, a_{22} y, a_{33} z\right)+$ higher order terms and $a_{33}^{2}=a_{11} a_{22}$

(2) $\left(\psi_{1}, \psi_{2}, \psi_{3}\right)=\left(a_{12} y, a_{21} x, a_{33} z\right)+$ higher order terms and $a_{33}^{2}=a_{12} a_{21}$.

Recall that the Bergman function $B_{V}=\left\|\phi_{00}\right\|_{M}^{2} \Theta_{V}-\left\|\phi_{00}\right\|_{M}^{4} \Theta_{V}^{2}+\cdots$. where $M$ is the minimal resolution of $V$ as described in section 3 , and $\Theta_{V}$ is given by (3.4). In view of Theorem 2.8, we have

$$
B_{V}((x, y, z), \overline{(x, y, z)})=B_{V}(\Psi(x, y, z), \overline{\Psi(x, y, z)}) .
$$

Putting (1) and (2) in (5.1) and comparing the 3rd order terms in (5.1), we see easily the 2 nd order terms of $\left(\psi_{1}, \psi_{2}, \psi_{3}\right)$ are zero. Repeating this argument, we see that $\left(\psi_{1}, \psi_{2}, \psi_{3}\right)$ has only linear terms. Using (5.1) again, we obtain $\left|a_{11}\right|=\left|a_{22}\right|=\left|a_{12}\right|=\left|a_{21}\right|=1$.

In Corollary 3.8, we need to assume that our invariant $\nu_{X}$ is not $\frac{1}{2}$. If we consider only automorphism instead of biholomorphism, we can deal with the case $\nu_{X}=\frac{1}{2}$.

Theorem 5.2. Let $V=\left\{(x, y, z) \in \mathbb{C}^{3}: x y=z^{2}\right.$ and $\eta(x, y, z)<\epsilon_{0}$, where $\eta$ is a strictly plurisubharmonic Reinhardt function $\}$. Suppose that $\nu_{X}=\frac{1}{2}$ where $\nu_{X}$ is the CR invariant of $X=\partial V$ defined in Theorem 3.6. Then the automorphism $\psi=\left(\psi_{1}, \psi_{2}, \psi_{3}\right): X \rightarrow X$ must be one of the following forms:

(1) $\left(\psi_{1}, \psi_{2}, \psi_{3}\right)=\left(e^{i \theta_{1}} x, e^{i \theta_{2}} y, e^{i \frac{\theta_{1}+\theta_{2}}{2}} z\right)$

(2) $\left(\psi_{1}, \psi_{2}, \psi_{3}\right)=\left(e^{i \theta_{1}} y, e^{i \theta_{2}} x, e^{i \frac{\theta_{1}+\theta_{2}}{2}} z\right)$

(3) $\left(\begin{array}{c}\psi_{1} \\ \psi_{2} \\ \psi_{3}\end{array}\right)=\left(\begin{array}{ccc}\alpha r e^{-i \theta} a_{31} & \frac{1}{r} e^{-i \theta} a_{32} & a_{13} \\ \frac{1}{\alpha r} e^{i \theta} a_{31} & r e^{i \theta} a_{32} & \frac{1}{\alpha} e^{2 i \theta} a_{13} \\ a_{31} & a_{32} & \frac{\alpha r^{2}+1}{2 \alpha r} e^{i \theta} a_{13}\end{array}\right)\left(\begin{array}{c}x \\ y \\ z\end{array}\right)+$ higher order terms

$$
\begin{aligned}
& \text { where } \alpha=\frac{-2\left\|\phi_{11}\right\|_{M}^{2}}{\left\|\phi_{12}\right\|_{M}^{2}} \\
& \qquad \begin{aligned}
a_{31} & =\frac{r}{|\alpha| r^{2}+1} e^{i \theta_{31}} \\
a_{32} & =\frac{r\left\|\phi_{10}\right\|_{M}}{\left\|\phi_{12}\right\|_{M}+r^{2}\left\|_{10}\right\|_{M}} e^{i \theta_{32}} \\
a_{13} & =\frac{2 r\left\|\phi_{10}\right\|_{M}}{r^{2}\left\|\phi_{10}\right\|_{M}+\left\|\phi_{12}\right\|_{M}} e^{i\left(\frac{\pi}{2}+\frac{\theta_{31}}{2}+\frac{\theta_{32}}{2}-\theta\right)} .
\end{aligned}
\end{aligned}
$$

Proof. The proof is the same as the proof of Theorem 3.7 except in case 1(b) where we did use the assumption $\nu_{X_{2}} \neq \frac{1}{2}$. Therefore to prove Theorem 5.2, it is sufficient to prove that statement (3) above occurs in case 1(b) of the proof 
of Theorem 3.7 under the assumption

$$
\nu_{X}=\frac{\left\|\phi_{11}\right\|_{M}^{2}}{\left\|\phi_{10}\right\|_{M}\left\|\phi_{12}\right\|_{M}}=\frac{1}{2} .
$$

Recall that in case 1(b) of the proof of Theorem 3.7, we have

$$
\begin{aligned}
a_{11}=r_{1} a_{31}, & a_{21}=\frac{1}{r_{1}} a_{31} \\
a_{22}=r_{2} a_{32}, & a_{12}=\frac{1}{r_{2}} a_{32} \\
a_{23}=\frac{r_{2}}{r_{1}} a_{13}, & a_{33}=\left(\frac{r_{2}}{2}+\frac{1}{2 r_{1}}\right) a_{13} \\
\alpha & =\frac{r_{1}}{\bar{r}_{2}}=\frac{-2\left\|\phi_{11}\right\|_{M}^{2}}{\left\|\phi_{12}\right\|_{M}^{2}}=\frac{-\left\|\phi_{10}\right\|_{M}}{\left\|\phi_{12}\right\|_{M}} .
\end{aligned}
$$

Let

$$
r_{2}=r e^{i \theta}
$$

Then

$$
r_{1}=\alpha r e^{-i \theta}=\frac{-\left\|\phi_{10}\right\|_{M}}{\left\|\phi_{12}\right\|_{M}} r e^{i \theta}
$$

and

$$
\left|r_{1}\right|=\frac{\left\|\phi_{10}\right\|_{M}}{\left\|\phi_{12}\right\|_{M}} r
$$

Putting (3.29), (3.30), (3.18) and (3.19) into (3.7), we get

$$
-a_{13}^{2}\left(\frac{r_{2}}{2}-\frac{1}{2 r_{1}}\right)^{2}+a_{31} a_{32}\left(r_{1} r_{2}+\frac{1}{r_{1} r_{2}}-2\right)=0 .
$$

Putting (3.18) into (3.12), we get

$$
\left|a_{31}\right|^{2}\left\|\phi_{00}\right\|_{M}^{2}\left[\frac{\left|r_{1}\right|^{2}}{\left\|\phi_{10}\right\|_{M}^{2}}+\frac{1}{\left\|\phi_{11}\right\|_{M}^{2}}+\frac{1}{\left|r_{1}\right|^{2}\left\|\phi_{12}\right\|_{M}^{2}}\right]=\frac{\left\|\phi_{00}\right\|_{M}^{2}}{\left\|\phi_{10}\right\|_{M}^{2}}
$$

(5.2) and (5.11) imply

$$
\left|a_{31}\right|=\frac{\left|r_{1}\right|\left\|\phi_{12}\right\|_{M}}{\left|r_{1}\right|^{2}\left\|\phi_{12}\right\|_{M}+\left\|\phi_{10}\right\|_{M}}=\frac{r\left\|\phi_{10}\right\|_{M}}{|\alpha| r^{2}\left\|\phi_{10}\right\|_{M}+\left\|\phi_{00}\right\|_{M}}=\frac{r}{|\alpha| r^{2}+1} .
$$

Putting (3.19) into (3.13), we get

$$
\left|a_{32}\right|=\frac{\left|r_{2}\right|\left\|\phi_{10}\right\|_{M}}{\left\|\phi_{12}\right\|_{M}+\left|r_{2}\right|^{2}\left\|\phi_{10}\right\|_{M}}=\frac{r\left\|\phi_{10}\right\|_{M}}{\left\|\phi_{12}\right\|_{M}+\left|r^{2}\right|\left\|\phi_{10}\right\|_{M}} .
$$

Putting (3.29) and (3.30) into (3.14), we get

$$
\begin{aligned}
\left|a_{13}\right|^{2} & =\frac{2\left|r_{1}\right|^{2}\left\|\phi_{10}\right\|_{M}\left\|\phi_{12}\right\|_{M}}{\left|r_{1}\right|^{2}\left\|\phi_{12}\right\|_{M}^{2}+\frac{1}{2}\left(r_{1} r_{2}+1\right)^{2}\left\|\phi_{12}\right\|_{M}\left\|\phi_{10}\right\|_{M}+\left|r_{2}\right|^{2}\left\|\phi_{10}\right\|_{M}^{2}} \\
& =\frac{4 r^{2}\left\|\phi_{10}\right\|_{M}^{2}}{\left(r^{2}\left\|\phi_{10}\right\|_{M}+\left\|\phi_{12}\right\|_{M}\right)^{2}} .
\end{aligned}
$$


This implies

$$
\left|a_{13}\right|=\frac{2 r^{2}\left\|\phi_{10}\right\|_{M}^{2}}{r^{2}\left\|\phi_{10}\right\|_{M}+\left\|\phi_{12}\right\|_{M}}
$$

(5.9) implies

$$
a_{13}^{2}=a_{31} a_{32} \frac{4 r_{1}}{r_{2}} .
$$

Putting (5.12), (5.13) and (5.14) into (5.16), we get

$$
\frac{4 r^{2}\left\|\phi_{10}\right\|_{M}^{2}}{\left(r^{2}\left\|\phi_{10}\right\|_{M}+\left\|\phi_{12}\right\|_{M}\right)^{2}} e^{2 i \theta_{13}}=\frac{r}{|\alpha| r^{2}+1} \frac{r\left\|\phi_{10}\right\|_{M}}{\left\|\phi_{12}\right\|_{M}+r^{2}\left\|\phi_{10}\right\|_{M}} e^{i\left(\theta_{31}+\theta_{32}\right)} 4 \frac{\alpha r e^{-i \theta}}{r e^{i \theta}} .
$$

It follows that

$$
e^{2 i \theta_{13}}=e^{-2 i \theta+i \pi+i\left(\theta_{31}+\theta_{32}\right)} .
$$

Hence

$$
\theta_{13}=\frac{\pi}{2}+\frac{\theta_{31}}{2}+\frac{\theta_{32}}{2}-\theta
$$

Corollary 5.3. Let $V=\left\{(x, y, z) \in \mathbb{C}^{3}: x y=z^{2}\right.$ and $\eta(x, y, z)<\epsilon_{0}$, where $\eta$ is a strictly plurisubharmonic Reinhardt function $\}$. Suppose that $\nu_{X}=\frac{1}{2}$ where $\nu_{X}$ is the CR invariant of $X=\partial V$ defined in Theorem 3.6. If case (3) of Theorem 5.2 occurs, then the CR automorphism group of $X$ contains a 4-dimensional linear subgroup of the following form:

$$
\left(\begin{array}{c}
\psi_{1} \\
\psi_{2} \\
\psi_{3}
\end{array}\right)=\left(\begin{array}{ccc}
\alpha r e^{-i \theta} a_{31} & \frac{1}{r} e^{-i \theta} a_{32} & a_{13} \\
\frac{1}{\alpha r} e^{i \theta} a_{31} & r e^{i \theta} a_{32} & \frac{1}{\alpha} e^{2 i \theta} a_{13} \\
a_{31} & a_{32} & \frac{\alpha r^{2}+1}{2 \alpha r} e^{i \theta} a_{13}
\end{array}\right)\left(\begin{array}{c}
x \\
y \\
z
\end{array}\right)
$$

where

$$
\begin{aligned}
\alpha & =\frac{-2\left\|\phi_{11}\right\|_{M}^{2}}{\left\|\phi_{12}\right\|_{M}^{2}} \\
a_{31} & =\frac{r}{|\alpha| r^{2}+1} e^{i \theta_{31}} \\
a_{32} & =\frac{r\left\|\phi_{10}\right\|_{M}}{\left\|\phi_{12}\right\|_{M}+r^{2}\left\|\phi_{10}\right\|_{M}} e^{i \theta_{32}} \\
a_{13} & =\frac{2 r\left\|\phi_{10}\right\|_{M}}{r^{2}\left\|\phi_{10}\right\|_{M}+\left\|\phi_{12}\right\|_{M}} e^{i\left(\frac{\pi}{2}+\frac{\theta_{31}}{2}+\frac{\theta_{32}}{2}-\theta\right)} .
\end{aligned}
$$

Now we are ready to compute the automorphism group of $X$ even if $\nu_{X}=\frac{1}{2}$.

Theorem 5.4. Let $X_{a}=\left\{(x, y, z) \in \mathbb{C}^{3}: a|x|^{2}+|y|^{2}+|z|^{2}=\epsilon_{0}\right\}$ and $\nu_{a}$ be the $C R$ invariant defined in the previous section. Then $\nu_{1 / 4}=\nu\left(X_{1 / 4}\right)=\frac{1}{2}$ and the automorphism group of $X_{1 / 4}$ consists of biholomorphic map $\Psi=\left(\psi_{1}, \psi_{2}, \psi_{3}\right)$ of the following forms: 
(1) $\left(\psi_{1}, \psi_{2}, \psi_{3}\right)=\left(e^{i \theta_{1}} x, e^{i \theta_{2}} y, e^{i \frac{\theta_{1}+\theta_{2}}{2}} z\right)$

(2) $\left(\psi_{1}, \psi_{2}, \psi_{3}\right)=\left(e^{i \theta_{1}} y, e^{i \theta_{2}} x, e^{i \frac{\theta_{1}+\theta_{2}}{2}} z\right)$.

Proof. We only need to prove that the automorphism group of $X_{1 / 4}$ does not have element of the form in Corollary 5.3. Suppose on the contrary that automorphism of the form in Corollary 5.3 does exist. Since $\left(0, \sqrt{\epsilon_{0}}, 0\right)$ is in $X_{1 / 4}$, we have $\left(\frac{\sqrt{\epsilon_{0}}}{r} e^{-i \theta} a_{32}, \sqrt{\epsilon_{0}} r e^{i \theta} a_{32}, \sqrt{\epsilon_{0}} a_{32}\right)$ in $X_{1 / 4}$. Hence

$$
\frac{1}{4} \frac{\epsilon_{0}}{r^{2}}\left|a_{32}\right|^{2}+\epsilon_{0} r^{2}\left|a_{32}\right|^{2}+\epsilon_{0}\left|a_{32}\right|^{2}=\epsilon_{0}
$$

This implies

$$
\left(\frac{1}{4 r^{2}}+r^{2}+1\right) \frac{r^{2}\left\|\phi_{10}\right\|_{M}^{2}}{\left(\left\|\phi_{12}\right\|_{M}+r^{2}\left\|\phi_{10}\right\|_{M}^{2}\right.}=1
$$

It follows that

$$
r^{2}=\frac{\left\|\phi_{10}\right\|_{M}+2\left\|\phi_{12}\right\|_{M}}{4\left\|\phi_{10}\right\|_{M}}
$$

This is absurd.

\section{References}

[An-Gr] A. Andreotti and H. Grauert, Theéorémes de finitude pour la cohomologic des espaces complexes, Bull. Soc. Math. France 90 (1962), 193-259.

[Bo] L. Boutet De Monvel, Integration des equation de Cauchy-Riemann, Seminaire Goulaouic-Lions-Schwartz, Ex ré IX, 1974-1975.

[Ca] E. Cartan, Sur la geometric pseudo-conforme des hypersurfaces de l'espace de deux variables complex. I and II, Ann. Mat. Pura Appl. 11 (1932), 17-90, and Ann. Scuola Norm. Sup. Pisa Sci. Fis, Mat. 1 (1932), 333-354.

[Ch-Sh] S.-C. Chen and M.-C. Shaw, Partial Differential Equations in Several Complex Variables, AMS/IP, Studies in Advanced Mathematics, 19, 2001.

[Ch-Mo] S.S. Chern and J. Moser, Real hypersurfaces in complex manifolds, Acta Math. 133, (1974), 219-271.

[Fe] C. Fefferman, The Bergman kernel and biholomorphic mappings of pseudoconvex domains, Invent. Math. 26 (1974), 1-65.

[Ha-La] R. Harvey and B. Lawson, On boundaries of complex analytic varieties I, Ann. of Math. 102 (1975), 233-290.

[Hi] H. Hironaka, Resolution of singularities of an algebraic variety over a field of characteristic zero, Ann. of Math. 79, (1964), 109-326.

[Ko] S. Kobayashi, Geometry of bounded domains, Trans. Amer. Math. Soc. 92 (1959), 267-290.

[Koh] J. Kohn, The range of the tangential Cauchy-Riemann operator, Duke Math. J., 53 (1986), 525-545.

[La] H. Laufer, On rational singularities, Amer. J. Math. 94 (1972), 597-608.

[L-Y-Y] H.-S. Luk, S.S.-T. Yau and L.-Y. Leh, Bergman kernels on resolutions of isolated singularities, Mathematical Research Letters, 8 (2001), 303-319.

[We] S.M. Webster, Pseudo-Hermitian structures on a real hypersurface, Journal of Differential Geometry, 13, (1978), 25-41.

[Ya] S.S.-T. Yau, Two theorems on higher dimensional singularities, Math. Ann. 231 (1977), 55-59. 
Department of Mathematics, Statistics and Computer Science (M/C 249), University of Illinois at Chicago, 851 South Morgan Street, Chicago, IL 60607-7045, USA

E-mail address: yau@uic.edu

Institute of Mathematics, East China Normal University, Shanghai, China 